his friends advised his calling upon me on the following morning.

On Tuesday, February 20th, I first saw him amongst my other gratuitous patients. He complained of rheumatic pain in the fore-arm and shoulder, said he had headach, and that he could not eat, for nothing seemed to pass that part, drawing his hand across the abdomen a little above the umbilicus. His pulse was soft, and about 70 in the minute, and his tongue moist, and slightly furred; his manner was hurried, and the expression of his countenance nervous; his bowels had not been opened since the previous morning. I regarded it as a case af dyspepsia, and prescribed some aperient and antacid medicine, with a little tincture of hop.

At about seven o'clock in the evening of the same day I was called upon by his sister-in-law and requested to see him. She stated that he had been delirious two or three times in the course of the day, that he could neither eat nor drink, and that, although she had not thought of it before, a friend whom she met on her way here had reminded her of the bite, and had inquired if that was not the cause of his illness.

I was with him in about half an hour, and found him seated in a chair, in his brother's house, with a countenance expressive of horror, and complaining much of pain in the lower part of the chest and upper part of the abdomen, in the region of the diaphragm.

I desired him to swallow some tea, which was brought to him in a cup. He took it in his hand, settled his countenance, as if about to make a great effort, then gulped it down, without losing a drop, and started back convulsively two or three paces. His pulse was soft, feeble, small, and 70 or 72 in the minute; his tongue moist, and nearly free from fur; his respiration hurried, and every now and then convulsive jerking. I listened to the back of the chest and found the air everywhere entering the pulmonary vesicles. His bowels had not been opened for about thirty-six hours. He coughed hoarsely every few minutes, and the sound was similar to that of a child in the first stage of croup, not unlike the bark of a dog.

I prescribed calomel, extract of hyoscyamus, extract of colocynth, of each three grains; croton oil, half a drop make into two pills, to be taken every third hour, until the bowels are freely moved. And then extract of belladonna, one grain ; hydrocyanic acid, one scruple; syrup of poppies, half an ounce; camphor mixture, three ounces; cinnamon-water, two ounces and a half; the sixth part to be taken every three hours after the pills had operated.

It has been before remarked, that he had been a little delirious two or three times in the course of the day. He now became worse, and this rapidly. His delirium was more furious, and he would have injured those around him, and perhaps destroyed himself, for he endeavoured to get at the poker apparently for that purpose, until at about five in the morning he was secured in a straightwaistcoat. He had repeated vomitings during the night, and expectorated a great deal, spitting all about the room, and upon the hands of those around him, as if purposely.

During the night, owing, it is supposed, to the directions having been misunderstood, he took nearly the whole of his medicine mixture as well as pills, notwithstanding which the bowels did not act. It was observed that the symptoms were worse about every two hours, when they diminished somewhat until the next period returned. The poor fellow expired about eight o'clock on Wednesday morning, the 24 th of February, a day less than nine weeks from the time he was bitten, and three days from the first accession of pain in the hand.

Autopsu. - The examination of the body (by direction of the coroner for Middlesex) was made about forty-seven hours after death, the weather being very cold at the time.

External Appearances.-Slight lividity of face, groins, and beneath the nails of the toes, and considerable lividity of ears, back of the body and limbs, and beneath the nails of the hands. The hands were fixed in a semiflexed and semi-prone condition.

There was a good deal of congestion of the scalp and cranium. The sinuses were loaded with blood. The tunica arachnoides thickened and opake, slightly opaline. The pia mater greatly congested. The sections of the cerebrum presented numerous bloody points. The lateral ventricles contained about a drachm and a half of serum. The plexus choroides were gorged with blood. There were about six drachms of serum at the base of the brain.

The salivary glands were rather livid; the mucous membrane of the funces, epiglottis, larynx, trachea, and bronchi very vascular; and the air-passages, from the glottis downwards, filled with bloody froth, the bubbles of which were exceedingly small and regular. The lungs were everywhere permeable to air; the right lung was remarkably red, approaching to a vermilion colour, whilst the left was nearly as dark as the colour of venous blood. The pericardium had its two surfaces intimately united by old adhesions, which had become organised, so that, practically speaking, he had no pericardium. This was, probably, owing to an attack of acute rheumatism five years ago, which confined him for three months. The heart itself appeared to be healthy, but all its cavities contained very dark blood, scarcely any of which was coagulated, and the little coagulum there was, was even firmer than currant-jelly, and homogeneous throughout.

The liver had a little of the nutmeg appearance. He had formerly been a free drinker, and this organ, as well as all the other" viscera of the abdomen, was congested. The gall-bladder was full, the urinary-bladder empty, as was also the stomach, which did not even contain any flatus. The intestines were moderately empty.

The fore-finger of the left hand, which was the part bitten, did not present any morbid appearance whatever. The marks of the wound in the skin were barely discernille, and there was not even the slightest hardness in the eicatrix. No mischief was observed in the course of the absorbent vessels, or in the glands of the axilla.

Remurhs-As the treatment for hydrophobia is so generally unsuccessful, notwithstanding the boldness with which bleeding is practised, I determined not to adopt that measure with a pulse which was decidedly feeble, and in the entire absence of any proof of inflammatory action. I should, however, were a similar case again presented to me, recommend moderate bleedings, as derivatives, to relieve the marked congestion of the whole system presented after death.

It has been my lot to open many hundred bodies since entering the profession, and if asked what constituted the marked peculiurities in the post-mortem appearances in this case-such appearances as I have not again and again witnessed in other diseases-I should refer to those presented by the pulmonary apparatus more especially. I have never witnessed such froth in the air-passages, nor such redness as the right lung presented. The bloody froth was of a bright-red colour, perfectly homogeneous, and apparently secreted of that colour, and not formed by the mixing of blood and thin mucus together, for, if so, it is scarcely probable that there would have been no difference in its appearance in different parts of the air-passages,-no difference was, however, anywhere observed.

The man Doddington, who was bitten in the left under eyelid, called upon me one evening, but as I could not then discern the slightest mark of the former wound, I desired him to see me by daylight. This he has not yet done.

Church-row, Islington, March 6, 1844.

\section{CASE OF LARGE OVARIAN TUMOUR REMOVED} BY OPERATION.

By Frederic Birn, M.D., \&c.

\section{[Read before the Medrcal Soctery of Lowdon, March 4, 1844.]}

THE subject of the case was a lady, on whom he had lately operated for the extirpation of a large ovarian tumour. The operation, although attended by unusual difficulties, had been completely successful. He had been induced to bring the case before the notice of the society, partly on account of the peculiar features it presented, and partly because it afforded a marked illustration of the imperfect state of the means of diagnosis of certain forms 
of ovarian disease. The subject of the case was thirtyfive years of age, married, but without children, and, with the exception of dysmenorrhæa, had enjoyed previous good health. During the last two years the abdomen had been increasing in size, the enlargement having taken place equally on either side, and had been, until within the last six months, quite unaccompanied by disturbance of the general health. Pregnancy was for some time supposed to exist, and, under that impression, no recourse was had, until lately, to remedial measures. The abdomen had, within the preceding four or five months, enlarged much more rapidly than before, frequent vomiting and protracted diarrhoa then occurred, and general emaciation succeeded. Two months ago, Dr. Frederic Bird saw the patient, in consultation with Mr. Hale Thomson and other centlemen, at which period the abdomen had a circumference of forty inches; fluctuation appeared very distinct in every direction; the thoracic cavity was much encroached upon by the large size of the tumour, she was greatly emaciated, and it was evident that the constitutional powers were fast sinking beneath the disease. Dr. Locock, Dr. Hamilton Roe, Dr. Hodgkin, Mr. B. Phillips, and others, subsequently saw the patient, and the operation for extirpation was finally determined upon.

The same preliminary treatment adopted in his former operations having been employed, Dr. F. Bird commenced by making a small incision in the linea alba, and a little below the umbilicus, and on exposing the peritoneum, the cyst was found to be adherent; the adhesions were then examined, and Dr. Locock concurring in the opinion that they would admit of separation without any great difficulty, the incision was enlarged to about five inches, so as to readily admit of the passage of the hand, which was next cautiously introduced between the surface of the tumour and the parietes of the abdomen; the adhesions were found to exist in every direction anteriorly, but, excepting in some few parts, gave way readily to the presence of the fingers; all the adhesions having been thus detached, and it having been previously found that the contents of the cyst were not fluid, an incision was made into it, and its bulk considerably reduced by the withdrawal of several pounds of the firm gelatinous mass by which it was fllled, and as soon as the tumour began to protrude from the wound, it was firmly grasped by the forceps, the incision carried upwards to about three inches, and the remaining part of the morbid growth removed from the cavity of the abdomen; the wound was then closed, and secured by sutures, the vessels of the pedicle having been previously tied and divided, and the ligatures fixed at the lower end of the incision. But little hæmorrhage occurred, and the operation was borne remarkably well by the patient, her pulse, at its conclusion, exceeding but by two beats the frequency observed during several days prior to its performance.

No pain, or other local symptom, was felt after the operation; reaction soon appeared, and as quickly subsided ; the patient passed a good night, and at the end of a few days had quitted her bed; the wound rapidly healed, and all the ligatures were removed before the end of the fourth week after the operation. The patient's convalescence had not been retarded by any subsequent symptoms, and she is now in complete health.

The tumour weighed thirty-five pounds. It consisted of the right ovarium, enlarged by the development of one large primary and several secondary cysts. The parent cyst was filled by a firm gelatinous secretion, varying in colour and in density ; the difference in colour being apparently due to the amount of blood sent to its several parts, the deepest colour being observed at the lowest portion of the mass. In some parts it was opake and striated. There were several vessels of large size traversing the interior of the tumour. The pedicle contained three arteries, of which one was large; the contents of the secondary cysts did not essentially differ from that contained in the primary one. The external surface of the tumour was irregularly covered by false membrane, which, in some parts, was of considerable density and firmness.

In making some remarks upon the preceding case, Dr. F. Bird said, the attendant circumstances of the opera- tion, in the present instance, had confirmed him in his opinion of the advantages to be gained by the employment of an incision of mediate size; the separation of the adhesions had, in this case, been found neither a tedious nor difficult proceeding, for the tense condition of the abdominal walls not having been destroyed by the large abdominal section, the hand was no sooner introduced laterally between the parietes of the abdomen and the contained tumour, than the adhesions were put upon the stretch, and, in that state, readily gave way before the presence of the fingers. Had the abdominal walls been more extensively divided, the detachment of the adhesions would have been a more difficull, and probably a more dangerous proceeding. Although the tumour was of large size, and did not contain any fluid, yet it was removed without having recourse to the very large incision. The history of the case had afforded no reason for believing that inflammation had occurred at any former period, and the adhesions were neither detected nor suspected. It was difficult to determine the period for which the peritoneal adhesions had existed, but the thickened, and in some parts well organised form of the false membrane, scattered over the surface of the tumour, seemed to indicate that they were not of very recent date. It was worthy of remark that, since the operation, the menstrual function had been twice performed, and on neither occasion had the patient experienced any of the severe pain from which she formerly suffered.

\section{REVIEWS,}

Elements of Natural Philosophy, being an Experimental Introduction to the Study of the Physical Sciences. By Golding BrRd, A.M., M.D., F.L.S., \&c. Second Edition. London. 8vo. Pp. 525.

A GENERAL knowledge of the principles of physical science is so indispensable to the medical student that every attempt to facilitate the attainment of such knowledge is at least meritorious in its object. Dr. Bird's work, though intended for general perusal, has been undertaken with especial reference to the wants of medical students. It is, on the whole, a very creditable production, and will be found useful to students of all classes; with reference, however, to its more immediate object, of introducing the student of medicine to a knowledge of natural philosophy, we find in it this defect-that, with a few exceptions, it gives no particular prominence to those points in which physics come most closely into apposition with medicine, and that, consequently, though decidedly nseful to the student of medicine, it is so only to an extent not much greater than that in which it would be useful to the student of any other science. Of this we shall presently notice instances. Dr. Bird's introduction to his work is neat and appropriate, though we think he has dwelt unnecessarily, both here and in chap.i., on the abstruse question of the intinite divisibility of matter. We also opine (in as far as it is possible to form an opinion on such a subject) that he has decided for the wrong side of this question. He denies that matter is infinitely divisible, but admits it to be "a most difficult task to adduce a precise refutation of all the mathematical sophistry and subtleties concerning infinite divisibility, with which the question has been loaded." Now, it appears to us that the sophistry and subtleties lie chiefly on our author's own side of the question, for, to our mind, the plainest way of viewing that question, and, indeed, the only way which is in any degree intelligible, is as follows :Matter, to be such, must be extended in the three dimensions of length, breadth, and thickness; if so, it must have several surfaces with intervening substance, or, if its figure be spherical, the spherical surface must bound a contained substance; consequently, if it were not for the intervening substance, the portions nearest the opposite surfaces, or, in 